\title{
Juggling COVID, work and family
}

IDA T. FONKOUE

Instructor of Medicine

Renal Division, Emory University School of Medicine

Atlanta, Georgia

DOI: $10.30824 / 2010-18$

If I could send a message to my pre-2020 self, I would say: Everything is not perfect, but these are great times, enjoy!

When I got the email in mid-march from my children's school that in-person instruction was immediately suspended until further notice, I shrugged and thought "oh well, that will give me a little break from getting up at 5:00 am every day to prepare breakfast, make the lunch my children will take to school and get them to the bus stop by 6:37 am". Fast forward 7 months later, research and participant recruitment have resumed on campus, but there is still no clear plan for when my children's school will resume in-person instruction. What a break!

For an early career scientist with no nanny, doing human research, who does not have the luxury of running experiments in the evening or at night, I find myself with the dilemma of prioritizing my research and work or prioritizing my children's instruction during this COVID-19 pandemic. In previous years, I believed that if I could work from home one or two days a week, I would be more productive. However, in 2020, I spent months working solely from home and yet struggled to stay productive. With the constant distraction of my children's instructional, nutritional and health needs, on top of their need for attention, it is hard to focus and complete any task requiring more than minimum brain cells. Juggling the daily zoom meetings while taking care of my children meant that the daytime work productivity was almost nil. Therefore, nighttime became the appointed time for serious and uninterrupted work. When I wasn't too exhausted and was able to be productive during those late hours, I paid the price of sleep deprivation the next day. As many parents, I normalized sleep deprivation over the years despite knowing its health consequences; but staying home because of COVID-19 created an additional anxiety-induced sleep deprivation. I am nevertheless privileged that my husband and I remain employed and are able to work from home and be present for our children during this pandemic. I don't take that for granted!

I learned a few things while juggling family and working from home because of COVID; some things that I wish I applied and some that I did. In retrospect, I realize that I didn't have to cook dinner every day. A few take out dinners a week could have freed some time for work or selfcare. My house didn't have to be tidy all the time. Nobody was visiting anyway! However, I was able to get my children to pick up chores and help with cleaning, dishes, laundry and more. I want to stress that every parent should find out and do what works best for their family in a situation like this. At the end of the day, it is better to have a sane mind and a messy house than an insane mind and a tidy house. A silver lining during this pandemic is how supportive and understanding students, colleagues, mentors and collaborators were (and still are) of the various interruptions and zoom bombing by children; who always happen to need something in a middle of a zoom meeting. I am truly grateful to the non-parents who have not made me feel incompetent or unprofessional during these unusual times we are still living in.

One particular challenge that parents like me who typically work outside the home have been facing, and for which we weren't prepared was the sudden fusion of three spaces that are normally separate in our daily life: the work space, the school space and the domestic space. Daily activities that take place in each space were suddenly all brought together to collide into the home/domestic space. I realized that finding a way to structure the house space and the routine, in order to minimize this fusion of spaces was very helpful. Depending on the size of your home, setting a separate space for kids' school and one for your work could minimize interferences and help improve productivity for all. To be honest, it didn't always work for us. 